TRANSACTIONS OF THE

AMERICAN MATHEMATICAL SOCIETY

Volume 363, Number 7, July 2011, Pages 3531-3552

S 0002-9947(2011)05120-2

Article electronically published on February 14, 2011

\title{
GENERATORS FOR RATIONAL LOOP GROUPS
}

\author{
NEIL DONALDSON, DANIEL FOX, AND OLIVER GOERTSCHES
}

\begin{abstract}
AвSTRACT. Uhlenbeck proved that a set of simple elements generates the group of rational loops in $\mathrm{GL}(n, \mathbb{C})$ that satisfy the $\mathrm{U}(n)$-reality condition. For an arbitrary complex reductive group, a choice of representation defines a notion of rationality and enables us to write a natural set of simple elements. Using these simple elements we prove generator theorems for the fundamental representations of the remaining neo-classical groups and most of their symmetric spaces. We also obtain explicit dressing and permutability formulae.
\end{abstract}

\section{Contents}

1. Introduction

2. The rational loop group associated to a representation 3533

3. Simple elements 3535

4. $\operatorname{CSp}(n, \mathbb{C}) \quad 3537$

4.1. Generating $\mathcal{L}\left(\operatorname{CSp}(n, \mathbb{C}), \mathbb{C}^{2 n}\right) \quad 3537$

4.2. Dressing and permutability $\quad 3539$

4.3. Generators for a family of twisted loop groups 3540

5. $\mathrm{SO}(n, \mathbb{C}) \quad 3541$

5.1. Generating $\mathcal{L}\left(\mathrm{SO}(n, \mathbb{C}), \mathbb{C}^{n}\right)$

5.2. Dressing and permutability 3542

5.3. Generators for the twisted loop groups 3543

6. $\mathbb{G}_{2} \quad 3544$

6.1. The linear algebra of $\mathbb{G}_{2}$ and its fundamental representation 3544

6.2. Generating $\mathcal{L}\left(\mathbb{G}, \mathbb{C}^{7}\right)$

6.3. Dressing and permutability 3548

6.4. Generators for the twisted loop group 3549

Appendix A. An explicit description of $\mathrm{G}_{2} \subset \mathrm{SO}(7)$

Acknowledgement 3551

References 3551

Received by the editors January 23, 2009 and, in revised form, March 31, 2009 and April 13, 2009.

2000 Mathematics Subject Classification. Primary 22E67, 37K25, 53C35.

Key words and phrases. Loop group, integrable system, submanifold geometry, generator theorem, simple element, neo-classical.

The third author was supported by the Max-Planck-Institut für Mathematik in Bonn and a DAAD-postdoctoral scholarship. He would like to thank the University of California, Irvine, and especially Chuu-Lian Terng, for their hospitality.

(C)2011 American Mathematical Society Reverts to public domain 28 years from publication 


\section{INTRODUCTION}

The work in this paper is centered around loop group dressing actions and their geometric applications; see Terng-Uhlenbeck [12]. Given a complex reductive Lie group $G$, the (holomorphic) loops into $G$ are holomorphic maps $g$ from open dense subsets of $\mathbb{C P}^{1}$ into $G$ (for the abstract theory of loop groups, see Pressley-Segal [10]). As is well known, the Birkhoff factorisation theorem yields a dressing action of negative loops $g_{-} \in \mathcal{L}_{-}(G)$ (holomorphic near $\infty$ with $g_{-}(\infty)=1$ ) on positive loops $g_{+} \in \mathcal{L}_{+}(G)$ (holomorphic on $\mathbb{C}$ ) as follows: given generic $g_{ \pm} \in \mathcal{L}_{ \pm}(G)$, there exist $\hat{g}_{ \pm} \in \mathcal{L}_{ \pm}(G)$ such that $g_{-} g_{+}=\hat{g}_{+} \hat{g}_{-}$; the dressing action of $g_{-}$on $g_{+}$is then defined by $g_{-} * g_{+}:=\hat{g}_{+}$.

Many examples of this setup appear throughout the literature, often with additional (reality or twisting) conditions on the loops: for example, the loops satisfying $\tau g(\lambda)=g(\bar{\lambda})$, where $\tau$ is some anti-holomorphic involution of $G$, form a subgroup $\mathcal{L}^{\tau}(G)$ and are said to satisfy a reality condition. In many examples smooth maps into the positive loop group are seen to correspond to special submanifolds or to solutions to a system of PDEs. These are preserved under dressing transforms by negative loops, and so the transforms descend to transforms of submanifolds and solutions of PDEs. For example Uhlenbeck [14] applies this construction to harmonic maps into $\mathrm{U}(n)$; Terng's group [12, 1, 6] studies her $U / K$-systems [1]; other applications include 4, 8, 2, No explicit formula for the dressing action of a generic negative rational loop is known to exist, though for any particular element it may be obtained using residue calculus. Most of the authors cited above make use of special negative loops, termed simple factors or simple elements, the dressing action of which does admit a simple formula.

Examples of simple factors typically exhibit several of the following desirable properties:

(1) They are rational loops with as few poles as possible, of lowest possible degrees.

(2) Their dressing action is explicitly calculable.

(3) They act by (simple) geometric transforms on any underlying geometry of the Lie group.

(4) There is a permutability theorem: given simple factors $p_{1}, p_{2}$ with pole sets $D_{1}, D_{2}$, there should exist simple factors $\hat{p}_{1}, \hat{p}_{2}$ with pole sets $D_{1}, D_{2}$ such that $\hat{p}_{1} p_{2}=\hat{p}_{2} p_{1}$; the dressing transforms of each side therefore correspond.

(5) The collection of simple factors generates the rational loop group.

The above list describes the most desirable situation. However, the fifth point has only ever been demonstrated for $\mathrm{U}(n)$ [14] and for the twisted loop group associated to $\mathrm{U}(n) / \mathrm{O}(n)$ [13. (More recently, a unique factorisation theorem for $\mathrm{U}(n)$ was obtained by Dai-Terng [5.) In the examples we consider, we show that the general notion of a simple factor introduced in Definition 3.2 produces elements that satisfy properties (11), (2), (4), and (5). We do not study the corresponding geometric transformations.

As was noted by Pressley-Segal [10, rationality of loops is only defined for matrix groups. More formally, in Section 2 we define rationality for any group $G$ with respect to any representation: $g \in \mathcal{L}(G)$ is rational with respect to a representation $\rho: G \rightarrow \operatorname{End}(V)$ iff $\rho \circ g$ is rational. In the literature, authors have generally 
used the adjoint representation or, when dealing with matrix groups, the standard matrix representation.

In Section 3 we define simple factors for any representation of a complex reductive Lie group that satisfies the reality condition with respect to a compact real form. The simple factors depend on the chosen representation. The various definitions of a simple factor given in the papers above are all special cases of the definition presented in Section 3 ,

In Sections 4, 5, and 6 we prove generating theorems for the rational loop groups of the fundamental representations of $\mathrm{SO}(n, \mathbb{C}), \mathbb{G}_{2}$, and the conformal symplectic group $\operatorname{CSp}(n, \mathbb{C})$. Together with Uhlenbeck's work this establishes generating theorems for the fundamental representations of all of the neo-classical groups. The appearance of the conformal symplectic group may seem strange at first, but we needed the conformal freedom in order to prove a generating theorem for the simple factors of Definition 3.2. Since centres of groups act trivially through the dressing action, this is of no concern for geometric applications. Indeed this approach has been followed before: dressing of positive loops in $\operatorname{SL}(n, \mathbb{C})$ tends to be done instead by $\mathrm{GL}(n, \mathbb{C})$-simple factors, e.g. 12 . Rui Pacheco has indicated in a private communication that he has obtained generating theorems for $\mathrm{SO}(n), \mathrm{SU}(n)$, and $\operatorname{Sp}(n)$ using different sets of generators.

Our simple factors have the same general form in each situation: all are sums of projections onto certain weight spaces with rational functions as coefficients. For example, in $\operatorname{CSp}(n, \mathbb{C})$ we calculate that

$$
p_{\alpha, W}(\lambda)=\frac{\lambda-\alpha}{\lambda-\bar{\alpha}} \pi_{W}+\pi_{W^{\perp}}
$$

is a simple element, where $\pi_{W}$ is the projection onto a Lagrangian subspace $W \subset$ $\mathbb{C}^{2 n}$ and ${ }^{\perp}$ is the Hermitian perp. Although our generating theorems follow the same strategy that was used by Uhlenbeck in $\operatorname{GL}(n, \mathbb{C})$ - expand a rational loop in a power series about a pole and use simple elements to reduce the order of the pole - the linear algebra associated to each group and representation alters the nature of the details. The case of the fundamental representation of $\mathrm{G}_{2}$ is the most involved. Aside from cases such as $\mathrm{SO}(3)$, in which the adjoint representation is isomorphic to the standard representation, we do not have proofs for generating theorems for any representations other than the fundamental ones.

For the fundamental representation of each group we prove a permutability formula and explicit expressions for the dressing action of simple factors. In contrast to the classical groups, and as observed in [2, 3], 10, the dressing action and permutability formulae for $\mathbb{G}_{2}$ must depend on derivatives of the loop being dressed.

Applying generating theorems to submanifold geometries of symmetric spaces (for example, harmonic maps into symmetric spaces or the $U / K$-systems) requires generating theorems for twisted loop groups. We extend our generating theorems to the twisted loop groups associated to all symmetric spaces with $\mathrm{SO}(n)$ or $\mathrm{G}_{2}$ as their isometry group and to the symmetric space $\operatorname{CSp}(n) / \mathrm{U}(n)$. We do not currently see how to handle the complex and quaternionic Grassmannians.

\section{The RAtional loop Group Associated to A Representation}

Associated to every complex Lie group $G$ is its holomorphic loop group

$$
\mathcal{L}(G):=\left\{g: U \rightarrow G \text { holomorphic } \mid U \subset \mathbb{C P}^{1} \text { is open and dense }\right\} ;
$$


multiplication requires intersecting the respective domains. We do not distinguish between loops that agree on an open and dense set.

We are interested in subgroups of $\mathcal{L}(G)$ consisting of functions that are rational on $\mathbb{C P}^{1}$, but the notion of rationality is not defined when the target space is an arbitrary complex manifold. Already, Pressley-Segal [10] point out that the notion of a rational loop group only exists for matrix groups. So for any representation $\rho: G \rightarrow \mathrm{GL}(V)$, we can define the rational loop group associated to $\rho$ to be

$$
\mathcal{L}(G, \rho):=\left\{g \in \mathcal{L}(G) \mid \rho \circ g: \mathbb{C P}^{1} \rightarrow \operatorname{End}(V) \text { is meromorphic }\right\} .
$$

The notation $\mathcal{L}(G, V)$ will also be used.

\section{Examples 2.1.}

(1) Let $G=\mathbb{C}^{*}$ and $\rho_{k}: \mathbb{C}^{*} \rightarrow \mathrm{GL}(\mathbb{C}) ; z \mapsto z^{k}$. Then

$$
\mathcal{L}\left(\mathbb{C}^{*}, \rho_{k}\right):=\left\{g: \mathbb{C P}^{1} \rightarrow \mathbb{C} \mid g^{k} \text { is rational }\right\} .
$$

(2) $\mathcal{L}(G, 1)=\mathcal{L}(G)$, where 1 denotes the trivial representation.

(3) $\mathcal{L}(G, \rho \oplus \sigma)=\mathcal{L}(G, \rho) \cap \mathcal{L}(G, \sigma)$ for all representations $\rho$ and $\sigma$.

(4) Let $G=\mathrm{SL}(3, \mathbb{C})$. Then the loop

$$
\lambda \mapsto\left(\begin{array}{ccc}
\lambda^{\frac{1}{3}} & 0 & 0 \\
0 & \lambda^{\frac{1}{3}} & 0 \\
0 & 0 & \lambda^{-\frac{2}{3}}
\end{array}\right)
$$

is rational with respect to the adjoint representation, but not with respect to the standard representation.

Lemma 2.2. The loop $f \in \mathcal{L}(G)$ is meromorphic at $\lambda_{0}$ with respect to $\rho$ if and only if for all $v \in V$,

$$
\lambda \mapsto \rho(f(\lambda)) v
$$

is meromorphic at $\lambda_{0}$. In fact, it is enough to only consider a basis of $V$.

Let $G$ be connected complex reductive and $\tau$ an antiholomorphic involution on $G$ whose fixed point group $G^{\tau}$ is a compact real form of $G$. A loop $g$ is said to satisfy the reality condition with respect to $\tau$ if

$$
\tau(g(\bar{\lambda}))=g(\lambda) .
$$

If $\sigma$ is additionally a holomorphic involution on $G$ commuting with $\tau$ and $K=G^{\tau, \sigma}$ denotes the fixed point set of both $\tau$ and $\sigma$, then $G^{\tau} / K$ is a symmetric space. We say that $g$ is twisted with respect to $\sigma$ if

$$
\sigma(g(-\lambda))=g(\lambda) .
$$

A loop $g$ is negative if it is holomorphic at $\infty$ and $g(\infty)=e$; it is positive if it is holomorphic on all of $\mathbb{C}$. We use superscripts for the reality and twisting conditions, and subscripts for negativity and positivity. As before, the representation will occur in the notation to indicate rationality. For example, the group of negative rational loops satisfying both reality and twisting is denoted by $\mathcal{L}_{-}^{\tau, \sigma}(G, \rho)$ or $\mathcal{L}_{-}^{\tau, \sigma}(G, V)$. 


\section{Simple ELEMENTS}

As before, let $G$ be a connected complex reductive Lie group, i.e. the complexification of some compact real Lie group, and let $\mathfrak{g}$ be its Lie algebra. In the following we fix an antiholomorphic involution $\tau$ of $G$ giving rise to the compact real form $G^{\tau}$. Let $\rho: G \rightarrow \mathrm{GL}(V)$ be a representation. We assume that $\rho$ is almost faithful, i.e. has discrete kernel. Note that because $G$ is reductive, $\rho$ is completely reducible. In this section we formulate the notion of a simple element in $\mathcal{L}_{-}^{\tau}(G, \rho)$.

Definition 3.1. A semisimple element $H \in \mathfrak{g}$ is $\rho$-integral if $\rho(H) \in \operatorname{End}(V)$ has only integer eigenvalues.

Fix a maximal torus $T \subset G^{\tau}$ with Lie algebra $\mathfrak{t}$ and denote the complexification of $\mathfrak{t}$ by $\mathfrak{h} \subset \mathfrak{g}$. The elements of $\mathfrak{h}$ are semisimple and commute, and thus $\mathfrak{h}$ induces the decomposition

$$
V=\bigoplus_{\mu \in \Phi_{\rho}} V_{\mu}
$$

into simultaneous eigenspaces. For any $\mu \in \mathfrak{h}^{*}$ the subspace $V_{\mu} \subset V$ consists of those vectors $v$ such that $\rho(H) v=\mu(H) v$ for all $H \in \mathfrak{h}$. The indexing set $\Phi_{\rho} \subset \mathfrak{h}^{*}$ is the set of weights, i.e. those $\mu$ for which $V_{\mu}$ is nontrivial.

Recall that because $G^{\tau}$ is compact, there is a $G^{\tau}$-invariant hermitian inner product on $V$, with respect to which the elements of $\mathfrak{g}^{\tau}$ become skew-hermitian operators on $V$. Consequently, $\rho$-integral elements in $\mathfrak{h}$ are necessarily contained in the space it $\subset \mathfrak{g}$. Note that $H \in \mathfrak{h}$ is $\rho$-integral if and only if $\mu(H) \in \mathbb{Z}$ for all weights $\mu$. Using $\rho$-integral elements we can define simple elements, depending on the chosen representation:

Definition 3.2. For any $\alpha \in \mathbb{C} \backslash \mathbb{R}$ and $\rho$-integral $H \in i \mathfrak{g}^{\tau}$, the loop

$$
p_{\alpha, H}(\lambda)=\exp \left(\ln \left(\frac{\lambda-\alpha}{\lambda-\bar{\alpha}}\right) H\right)
$$

where ln denotes the natural logarithm, is a simple element.

The assumption that $H \in i \mathfrak{g}^{\tau}$ is needed since we want our simple elements to satisfy the $\tau$-reality condition:

Lemma 3.3. For any $\rho$-integral $H \in i \mathfrak{g}^{\tau}$ and $\alpha \in \mathbb{C} \backslash \mathbb{R}$, the simple element $p_{\alpha, H}$ satisfies the reality and normalization conditions.

Proof. Simple elements are obviously normalized at infinity. The reality condition is satisfied because

$$
\begin{aligned}
\tau\left(p_{\alpha, H}(\bar{\lambda})\right) & =\tau \exp \left(\ln \left(\frac{\bar{\lambda}-\alpha}{\bar{\lambda}-\bar{\alpha}}\right) H\right)=\exp \left(\overline{\left.\ln \left(\frac{\bar{\lambda}-\alpha}{\bar{\lambda}-\bar{\alpha}}\right) \tau(H)\right)}\right. \\
& =\exp \left(-\ln \left(\frac{\lambda-\bar{\alpha}}{\lambda-\alpha}\right) H\right)=p_{\alpha, H}(\lambda)
\end{aligned}
$$

where we used that $\tau(H)=-H$.

For the following lemma, let $\sigma$ be a holomorphic involution commuting with $\tau$ and $\mathfrak{g}^{\tau}=\mathfrak{k} \oplus \mathfrak{p}$ the corresponding Cartan decomposition; that is, $\mathfrak{k}$ is the +1 eigenspace of $\sigma$ and $\mathfrak{p}$ is the -1-eigenspace. 
Lemma 3.4. For any $\rho$-integral element $H \in i \mathfrak{p}$ and $r \in \mathbb{R} \backslash\{0\}$, the simple element $p_{i r, H}$ satisfies the $\sigma$-twisting condition.

Proof. Using $\sigma(H)=-H$ we calculate

$\sigma\left(p_{i r, H}(-\lambda)\right)=\exp \left(\ln \left(\frac{-\lambda-i r}{-\lambda+i r}\right) \sigma(H)\right)=\exp \left(-\ln \left(\frac{\lambda+i r}{\lambda-i r}\right) H\right)=p_{i r, H}(\lambda)$.

Simple elements with $H \in i$ act diagonally with respect to the $\mathfrak{t}$-weight decomposition:

Proposition 3.5. Let $H \in i$ t be $\rho$-integral. The action of the simple element $p_{\alpha, H}$ is given by

$$
\rho \circ p_{\alpha, H}(\lambda)=\sum_{\mu \in \Phi_{\rho}}\left(\frac{\lambda-\alpha}{\lambda-\bar{\alpha}}\right)^{\mu(H)} \pi_{V_{\mu}},
$$

where the $\pi_{V_{\mu}}$ are projections with respect to the decomposition (3.1).

Proof. For any weight vector $v \in V_{\mu}$,

$$
\begin{aligned}
p_{\alpha, H}(\lambda) \cdot v & =\exp \left(\ln \left(\frac{\lambda-\alpha}{\lambda-\bar{\alpha}}\right) H\right) v \\
& =\exp \left(\ln \left(\frac{\lambda-\alpha}{\lambda-\bar{\alpha}}\right) \mu(H)\right) v=\left(\frac{\lambda-\alpha}{\lambda-\bar{\alpha}}\right)^{\mu(H)} v .
\end{aligned}
$$

By Lemma 2.2, the loop $p_{\alpha, H}$ is rational with respect to $\rho$ because $\mu(H) \in \mathbb{Z}$.

Any $\rho$-integral element $H \in i \mathfrak{g}^{\tau}$ is $G^{\tau}$-conjugate to one in $i$ t. In particular, the proposition shows that simple elements are rational, i.e. $p_{\alpha, H} \in \mathcal{L}_{-}^{\tau}(G, \rho)$. Since $\rho$ is almost faithful, the $\rho$-integral elements in $i \mathfrak{t}$ form a lattice in $i$ t. If we choose an integral basis $H_{1}, \ldots, H_{r}$ for this lattice, any simple element of the form $p_{\alpha, H}$ with $H \in i \mathrm{t}$ is a product of the $p_{\alpha, H_{i}}$, since exp is a homomorphism on $\mathfrak{h}$.

Example 3.6. Let $G=\mathrm{GL}(n, \mathbb{C})$. The automorphism

$$
\tau(g)={ }^{*} g^{-1}
$$

(where ${ }^{*} g={ }^{t} \bar{g}$ ) gives rise to the compact real form $G^{\tau}=\mathrm{U}(n)$. Choose the maximal torus consisting of diagonal elements. The weights are $\mu_{1}, \ldots, \mu_{n}$, where

$$
\mu_{i}(X)=X_{i i}
$$

for any diagonal matrix $X$. The corresponding weight spaces are $L_{i}=\mathbb{C} \cdot e_{i}$, where $e_{1}, \ldots, e_{n}$ are the standard basis vectors of $\mathbb{C}^{n}$. Let $H_{i}$ be the diagonal matrices dual to the $\mu_{i}$. The $\left\{H_{i}\right\}$ form a basis of the lattice of $\rho$-integral elements. From Proposition 3.5 it follows that

$$
\rho \circ \exp \left(\ln \left(\frac{\lambda-\alpha}{\lambda-\bar{\alpha}}\right) H_{i}\right)=\left(\frac{\lambda-\alpha}{\lambda-\bar{\alpha}}\right) \pi_{L_{i}}+\pi_{K_{i}}=\operatorname{Id}+\frac{\bar{\alpha}-\alpha}{\lambda-\bar{\alpha}} \pi_{L_{i}},
$$

where $K_{i}=L_{1} \oplus \ldots \oplus \hat{L}_{i} \oplus \ldots \oplus L_{n}$, and the projections are defined with respect to the hermitian orthogonal decomposition of $\mathbb{C}^{n}$ into the $L_{j}$. Conjugating these with $\mathrm{U}(n)$, we see that the simple elements are loops of the form

$$
\mathrm{Id}+\frac{\bar{\alpha}-\alpha}{\lambda-\bar{\alpha}} \pi_{L}
$$


where $L \subset \mathbb{C}^{n}$ is any complex line and $\pi_{L}$ is the hermitian projection onto $L$. By multiplying several of these for which the complex lines are orthogonal, we obtain loops of the form

$$
\mathrm{Id}+\frac{\bar{\alpha}-\alpha}{\lambda-\bar{\alpha}} \pi_{W}
$$

where $\pi_{W}$ is the hermitian projection onto a complex subspace $W$; these are the simple elements introduced in [14].

Example 3.7. In the case that $\rho$ is the adjoint representation of a complex Lie group $G$ admitting parabolic subalgebras of height one, our simple elements generalise those considered in [7]. Grading elements, in particular canonical elements of pairs of complementary parabolic subalgebras, are Ad-integral.

Example 3.8. Consider a pair of representations $\rho_{1}: G \rightarrow \mathrm{GL}\left(V_{1}\right)$ and $\rho_{2}: G \rightarrow$ $\operatorname{GL}\left(V_{2}\right)$ such that 0 is a weight for $V_{1}$. Then the simple elements for $V_{1} \otimes V_{2}$ are a subset of those for $V_{2}$.

Example 3.9. Consider the case of $\mathrm{SL}(2, \mathbb{C})$. There is an irreducible representation $V_{n}$ of dimension $n$ and $V_{n} \subset S y m^{n-1}\left(\mathbb{C}^{2}\right)$. In this case the simple elements only depend on whether $n$ is odd or even.

We now turn our attention to the question of finding generators for the rational loop groups. In Sections 5.1 and 6.2 we will prove that the simple elements generate the rational loop groups $\mathcal{L}_{-}^{\tau}\left(\mathrm{SO}(n, \mathbb{C}), \mathbb{C}^{n}\right)$ and $\mathcal{L}_{-}^{\tau}\left(\mathbb{G}_{2}, \mathbb{C}^{7}\right)$. We think it plausible that the simple elements of Definition 3.2 generate the negative rational loop group whenever $\rho$ is the complexification of an orthogonal representation, i.e. $V=W \otimes \mathbb{C}$, $\rho\left(G^{\tau}\right) \subset \mathrm{O}(W)$ (and consequently $\left.\rho(G) \subset \mathrm{O}(V)\right)$. However, if the representation is not orthogonal, we believe the same statement will only be true if we pass to the extension by the centre of $\operatorname{GL}(V)$, i.e. the conformalization of $G$. For example, in Section 4.1 we show that the simple elements generate $\mathcal{L}_{-}^{\tau}\left(\operatorname{CSp}(n, \mathbb{C}), \mathbb{C}^{2 n}\right)$, where $\operatorname{CSp}(n)$ is the conformalization of $\operatorname{Sp}(n, \mathbb{C})$, but were unable to obtain a generating theorem for $\mathcal{L}_{-}^{\tau}\left(\operatorname{Sp}(n, \mathbb{C}), \mathbb{C}^{2 n}\right)$.

\section{4. $\operatorname{CSp}(n, \mathbb{C})$}

4.1. Generating $\mathcal{L}\left(\operatorname{CSp}(n, \mathbb{C}), \mathbb{C}^{2 n}\right)$. In this section, we prove that for the standard representation of $\operatorname{CSp}(n, \mathbb{C})$, which is $\mathbb{C}^{2 n}$ by our indexing, the simple elements defined in Section 3 generate the rational loop group $\mathcal{L}_{-}^{\tau}\left(\operatorname{CSp}(n, \mathbb{C}), \mathbb{C}^{2 n}\right)$.

Let $\omega$ be the standard symplectic form on $\mathbb{C}^{2 n}$. If

$$
J=\left(\begin{array}{cc}
0 & I_{n} \\
-I_{n} & 0
\end{array}\right),
$$

then

$$
\omega(v, w)={ }^{t} v J w
$$

The simple group $\operatorname{Sp}(n, \mathbb{C})$ is the group of (invertible) matrices that preserve $\omega$, and the conformalization of $\operatorname{Sp}(n, \mathbb{C})$ is the extended group

$$
\operatorname{CSp}(n, \mathbb{C})=\left\{A \in \mathrm{GL}(2 n, \mathbb{C}) \mid A^{*} \omega=c \cdot \omega \text { for some } c \in \mathbb{C}\right\} .
$$

We will make use of the standard hermitian inner product $(v, w)={ }^{t} \bar{v} w$ and the relation

$$
(v, w)=\omega(\bar{v}, J w)
$$


The Lie algebra of $\operatorname{CSp}(n, \mathbb{C})$ is

$$
\mathfrak{c} \mathfrak{s p}(n, \mathbb{C})=\left\{\left(\begin{array}{cc}
A & B \\
C & -{ }^{t} A
\end{array}\right)+a \cdot \operatorname{Id} \mid{ }^{t} B=B,{ }^{t} C=C, a \in \mathbb{C}\right\} .
$$

The involution $\tau(g)={ }^{*} g^{-1}$ of $\operatorname{CSp}(n, \mathbb{C})$ gives rise to the compact real form $\operatorname{CSp}(n)=\operatorname{Sp}(n) \times S^{1}$. It contains the $n+1$-dimensional torus consisting of diagonal matrices in $\operatorname{CSp}(n)$ whose complexification has the Lie algebra

$$
\mathfrak{h}=\left\{\left(\begin{array}{cc}
A & 0 \\
0 & -A
\end{array}\right)+a \cdot \mathrm{Id} \mid A \text { is diagonal, } a \in \mathbb{C}\right\} .
$$

The weights of the standard representation of $\operatorname{CSp}(n, \mathbb{C})$ on $\mathbb{C}^{2 n}$ are $\mu_{1}, \ldots, \mu_{2 n}$, where $\mu_{i}(X)=X_{i i}$, and the corresponding weight spaces are spanned by the standard basis elements $e_{1}, \ldots, e_{2 n}$.

Let $H=\left(\begin{array}{cc}I_{n} & 0 \\ 0 & 0\end{array}\right) \in \mathfrak{h}$. By Proposition 3.5 the simple element corresponding to the $\rho$-integral element $H$ is given by

$$
\exp \left(\ln \left(\frac{\lambda-\alpha}{\lambda-\bar{\alpha}}\right) H\right)=\left(\frac{\lambda-\alpha}{\lambda-\bar{\alpha}}\right) \pi_{W_{0}}+\pi_{W_{0}^{\perp}}
$$

where $W_{0}=e_{1} \wedge \ldots \wedge e_{n}$. Recall that an $n$-plane $W$ in $\left(\mathbb{C}^{2 n}, \omega\right)$ is Lagrangian if the symplectic form pulls back on it to be zero. Conjugating with $\operatorname{CSp}(n)$, we find that, for any Lagrangian subspace $W \subset \mathbb{C}^{2 n}$, the loop

$$
p_{\alpha, W}(\lambda)=\left(\frac{\lambda-\alpha}{\lambda-\bar{\alpha}}\right) \pi_{W}+\pi_{W^{\perp}}
$$

is a simple element. Note that the hermitian complement $W^{\perp}$ of $W$ is $J \bar{W}$.

Theorem 4.1. The simple elements $p_{\alpha, W}$, where $\alpha \in \mathbb{C} \backslash \mathbb{R}$ and $W \subset \mathbb{C}^{2 n}$ is a Lagrangian subspace, generate $\mathcal{L}_{-}^{\tau}\left(\operatorname{CSp}(n, \mathbb{C}), \mathbb{C}^{2 n}\right)$.

Proof. Let $g \in \mathcal{L}_{-}^{\tau}\left(\operatorname{CSp}(n, \mathbb{C}), \mathbb{C}^{2 n}\right)$ and fix a pole $\alpha \in \mathbb{C} \backslash \mathbb{R}$. We write the Laurant expansion of $g$ in $\frac{\lambda-\alpha}{\lambda-\bar{\alpha}}$ explicitly as

$$
g(\lambda)=\sum_{j=-k}^{\infty}\left(\frac{\lambda-\alpha}{\lambda-\bar{\alpha}}\right)^{j} g_{j},
$$

with $g_{-k} \neq 0$. The total degree of the pole at $\alpha$ is defined to be the pair $\left(k, \operatorname{rank} g_{-k}\right)$. We define $(k, n)<(l, m)$ if $k<l$ or $k=l$ and $n<m$. We proceed using induction on the total degree of each of the finitely many poles of the loop $g$. The reality condition $\tau(g(\bar{\lambda}))=g(\lambda)$ implies that if $g$ is holomorphic at $\alpha$ (i.e. it has no pole and is group valued), then it is also holomorphic at $\bar{\alpha}$. Our approach is to first remove the pole at $\alpha$ and then to modify the resulting loop so that it is holomorphic at $\alpha$ and thus also at $\bar{\alpha}$.

The fact that $g$ is a map into $\operatorname{CSp}(n, \mathbb{C})$ means that there is a $\mathbb{C}$-valued map $c_{g}$ satisfying

$$
\omega(g(\lambda) v, g(\lambda) w)=c_{g}(\lambda) \cdot \omega(v, w)
$$

for all $v, w \in \mathbb{C}^{2 n}$.

Inserting the power series expansion of $g$ into (4.3), we see that $c_{g}$ cannot have a pole at $\alpha$ of order higher than $2 k$. If $c_{g}$ has a pole of order exactly $2 k$, we get

$$
\omega\left(g_{-k} v, g_{-k} w\right)=c_{0} \cdot \omega(v, w)
$$


for some complex number $c_{0} \neq 0$. In particular, it follows that $g_{-k}$ is invertible, whereupon we form

$$
\tilde{g}=p_{\alpha, W} g
$$

for an arbitrary Lagrangian subspace $W$. Since the new $-k$-coefficient then has image only $W^{\perp}$, this reduces the total degree of the pole.

If $c$ has a pole of lower order at $\alpha$ (or none at all), (4.3) reduces to

$$
\omega\left(g_{-k} v, g_{-k} w\right)=0
$$

i.e. $\operatorname{Im} g_{-k}$ is $\omega$-isotropic. If we let $W$ be a Lagrangian subspace containing $\operatorname{Im} g_{-k}$, then the product $p_{\alpha, W} g$ has a pole at 0 of order at most $k-1$.

We continue this process until we obtain a loop (still referred to as $g$ ) that no longer has a pole at $\alpha$ and now show that it can be modified by simple factors so as to make it holomorphic at $\alpha$.

If $g_{0}=0$, we can certainly multiply with elements of the form $p_{\alpha, W}^{-1}$ until $g_{0} \neq 0$, so we may assume $g_{0} \neq 0$. As above, condition (4.3) then gives that $g_{0}$ is either invertible (in which case we are done) or that its image is $\omega$-isotropic.

In the second case, consider the map $\lambda \mapsto \operatorname{det}(g(\lambda))$. It is a polynomial in $\frac{\lambda-\alpha}{\lambda-\bar{\alpha}}$. Denote by $m$ the order of its zero at $\alpha$. Let $W$ be a Lagrangian subspace such that $\operatorname{Im} g_{0} \subset W^{\perp}$ and define $\tilde{g}=p_{\alpha, W}^{-1} g$. Then, $\tilde{g}$ has no pole at $\alpha$, its value $\tilde{g}(\alpha)$ is not zero, and the function

$$
\lambda \mapsto \operatorname{det}(\tilde{g}(\lambda))=\left(\frac{\lambda-\alpha}{\lambda-\bar{\alpha}}\right)^{-n} \operatorname{det}(g(\lambda))
$$

has a zero at $\alpha$ of order $m-n$. By induction, we can completely remove this zero and are left with a loop $g$ holomorphic at $\alpha$.

Once all poles are removed, we are left with a pole-free loop on $\mathbb{C P}^{1}$ with value Id at $\infty$. Liouville's theorem implies that this is the identity loop, and we are finished: $g$ has been written as a product of the generators.

4.2. Dressing and permutability. The following theorem and corollary are well known for the groups $\mathrm{GL}(n, \mathbb{C})[12,14$ and $\mathrm{SO}(n, \mathbb{C})$ [1, 2].

Theorem 4.2. Let $h \in \mathcal{L}(\operatorname{CSp}(n, \mathbb{C}))$ be holomorphic at $\alpha \in \mathbb{C} \backslash \mathbb{R}$. If we define $W^{\prime}=h(\alpha)^{-1} W$, then

is again holomorphic at $\alpha$.

$$
p_{\alpha, W} h p_{\alpha, W^{\prime}}^{-1}
$$

Proof. The compact group $\operatorname{CSp}(n)$ preserves the isotropic condition, so $W^{\prime}$ is also Lagrangian, and $p_{\alpha, W^{\prime}}$ is again a simple factor. We expand the new loop at $\alpha$ :

$$
p_{\alpha, W} h p_{\alpha, W^{\prime}}^{-1}(\lambda)=\left(\frac{\lambda-\bar{\alpha}}{\lambda-\alpha}\right) \pi_{W^{\perp}} h(\alpha) \pi_{W^{\prime}}+\ldots
$$

By our choice of $W^{\prime}$ the pole part vanishes, so it makes sense to evaluate the loop at $\alpha$. Since $\operatorname{CSp}(n, \mathbb{C})$ is closed in $\operatorname{GL}(n, \mathbb{C})$, it only remains to show that the endomorphism of $\mathbb{C}^{2 n}$ obtained by evaluating it at $\alpha$ is invertible. But its determinant is the same as the determinant of $h(\alpha)$, which is nonzero.

Corollary 4.3. Suppose that $p_{\alpha, W}$ and $p_{\beta, V}$ are simple factors such that $\alpha \neq \beta, \bar{\beta}$. Then

$$
p_{\beta, p_{\alpha, W}(\beta) V} p_{\alpha, W}=p_{\alpha, p_{\beta, V}(\alpha) W} p_{\beta, V}
$$

This follows from Theorem 4.2 in the standard way. 
4.3. Generators for a family of twisted loop groups. Let $\sigma$ be the holomorphic involution of $\operatorname{Sp}(n, \mathbb{C})$ defined by $\sigma(A)=J A J^{-1}$, where $J$ is defined in (4.1).

This gives rise to the symmetric space $\operatorname{Sp}(n) / \mathrm{U}(n)$. Extend $\sigma$ to an involution $\sigma_{c}$ on $\operatorname{CSp}(n, \mathbb{C})$ that acts on the center by $D \mapsto D^{-1}$. Also denote the derivative $\mathrm{d}\left(\sigma_{c}\right)_{1} \in \operatorname{Aut}(\mathfrak{c s p}(n, \mathbb{C}))$ by $\sigma_{c}$. While the fixed point set of $\sigma_{c}$ is again $\mathrm{U}(n)$, so that the respective symmetric spaces are different, the choice of a nontrivial extension of $\sigma$ aids the generator theorem. Recall that we say a loop $g$ is twisted, or satisfies the twisting condition, if

$$
\sigma_{c}(g(-\lambda))=g(\lambda)
$$

Lemma 4.4. If $\alpha \notin i \mathbb{R}$ and $p_{\alpha, W}$ is a simple element, then the product

$$
q_{\alpha, W}:=p_{-\alpha, p_{\alpha, W}(-\alpha) s W} p_{\alpha, W}=p_{\alpha, p_{-\alpha, s W}(\alpha) W} p_{-\alpha, s W}
$$

satisfies the twisting condition.

The proof follows immediately from the permutability proposition.

Theorem 4.5. The elements

$$
\begin{gathered}
p_{\alpha, W}(\lambda)=\left(\frac{\lambda-\alpha}{\lambda-\bar{\alpha}}\right) \pi_{W}+\pi_{W^{\perp}} \text {, where Wis a real Lagrangian, and } \alpha \in i \mathbb{R}, \\
\text { and } q_{\alpha, W}(\lambda) \text {, where } W \text { is any Lagrangian, and } \alpha \in \mathbb{C} \backslash(\mathbb{R} \cup i \mathbb{R})
\end{gathered}
$$

generate the twisted loop group $\mathcal{L}_{-}^{\tau, \sigma}\left(\operatorname{CSp}(n, \mathbb{C}), \mathbb{C}^{2 n}\right)$.

Proof. Let $g \in \mathcal{L}_{-}^{\tau, \sigma}\left(\operatorname{CSp}(n, \mathbb{C}), \mathbb{C}^{2 n}\right)$. We deal with the purely imaginary and nonimaginary poles of $g$ separately, as in the generating theorem for $\mathrm{U}(n) / \mathrm{O}(n)$ [13. First assume that $\alpha$ is a nonimaginary pole. By Theorem 4.1, we can find a Lagrangian $W$ such that $p_{\alpha, W} g$ has a pole of lower degree than $g$. Then, $q_{\alpha, W} g$ does so, too, and also satisfies the twisting condition. In this way, we remove the pole at $\alpha$, and the possibly occurring zero can then be dealt with in the same manner. In this way we can remove all of the nonimaginary poles.

Assume that $\alpha=i r$ is a purely imaginary pole of $g$, and write its power series expansion around $\alpha$ as

$$
g(\lambda)=\sum_{j=-k}^{\infty}\left(\frac{\lambda-i r}{\lambda+i r}\right)^{j} g_{j}
$$

with $g_{-k} \neq 0$. We want to show that $V=\operatorname{Im} g_{-k}$ is contained in a real Lagrangian subspace. As before, because $g$ is a loop in $\operatorname{CSp}(n, \mathbb{C})$, the image of $g_{-k}$ is either equal to $\mathbb{C}^{2 n}$ or $\omega$-isotropic. We get additional information by combining the reality and twisting conditions. They read

$$
g(\lambda)={ }^{*} g(\bar{\lambda})^{-1} \text { and } g(\lambda)=-J g(-\lambda) J
$$

and imply that

$$
(g(-\bar{\lambda}) v, J g(\lambda) J w)=-(v, w) .
$$

Expanding this as a power series, the lowest order term gives us

$$
\left(g_{-k} v, J g_{-k} J w\right)=0
$$

for all $v$ and $w$, i.e. $V \perp J V$. In particular, $V$ cannot be equal to $\mathbb{C}^{2 n}$, so it is also $\omega$-isotropic. Therefore, $\omega(\bar{V}, V)=(V, J V)=0$, i.e. $V$ is contained in the real isotropic space $V+\bar{V}$, which in turn is contained in some real Lagrangian $W$. We can therefore proceed as in Theorem 4.1 using simple factors corresponding to such $W$ : first we remove the pole, and then any possible resulting zero. 


\section{5. $\mathrm{SO}(n, \mathbb{C})$}

5.1. Generating $\mathcal{L}\left(\mathrm{SO}(n, \mathbb{C}), \mathbb{C}^{n}\right)$. In this section, we prove that for the standard representation of $\mathrm{SO}(n, \mathbb{C})$, the simple elements defined in Section 3 generate the rational loop group $\mathcal{L}_{-}^{\tau}\left(\mathrm{SO}(n, \mathbb{C}), \mathbb{C}^{n}\right)$. The compact real form $\mathrm{SO}(n)$ is given by the involution $\tau(A)=\bar{A}$. Let $\mathfrak{t} \subset \mathfrak{s o}(n)$ be a maximal abelian subalgebra with complexification $\mathfrak{h} \subset \mathfrak{s o}(n, \mathbb{C})$, and let $\pm \mu_{1}, \ldots, \pm \mu_{r}$ be the nonzero weights of the standard representation, where $r$ is the rank of $\mathrm{SO}(n)$. Note that $\mu_{1}, \ldots, \mu_{r}$ is a basis of $\mathfrak{h}^{*}$ and that zero is a weight if and only if $n$ is odd. Let $L_{i}$ be the weight space corresponding to $\mu_{i}$; then $\overline{L_{i}}$ is the weight space of $-\mu_{i}$. Both $L_{i}$ and $\overline{L_{i}}$ are isotropic lines in $\mathbb{C}^{n}$. The weight space decomposition is

$$
\mathbb{C}^{n}=V_{0} \oplus \bigoplus_{i=1}^{r}\left(L_{i} \oplus \overline{L_{i}}\right)
$$

Here $V_{0}$ is the weight space corresponding to the weight 0 , if $n$ is odd, and is empty otherwise. Let $H_{i}$ be such that $\mu_{j}\left(H_{i}\right)=\delta_{i j}$. The $H_{i}$ form a basis of the $\rho$-integral lattice, and by Proposition 3.5 we have

$$
\exp \left(\ln \left(\frac{\lambda-\alpha}{\lambda-\bar{\alpha}}\right) H_{i}\right)=\left(\frac{\lambda-\bar{\alpha}}{\lambda-\alpha}\right) \pi_{\overline{L_{i}}}+\pi_{\left(L_{i} \oplus \overline{L_{i}}\right)^{\perp}}+\left(\frac{\lambda-\alpha}{\lambda-\bar{\alpha}}\right) \pi_{L_{i}} .
$$

Conjugating $H_{i}$ with elements of $\mathrm{SO}(n)$, we find that the loops

$$
p_{\alpha, L}=\left(\frac{\lambda-\bar{\alpha}}{\lambda-\alpha}\right) \pi_{\bar{L}}+\pi_{(L \oplus \bar{L})^{\perp}}+\left(\frac{\lambda-\alpha}{\lambda-\bar{\alpha}}\right) \pi_{L},
$$

where $L$ is an arbitrary isotropic line in $\mathbb{C}^{n}$, are simple elements in $\mathcal{L}_{-}^{\tau}(\mathrm{SO}(n, \mathbb{C}), \mathbb{C})$.

Theorem 5.1. The simple elements $p_{\alpha, L}$, where $\alpha \in \mathbb{C} \backslash \mathbb{R}$ and $L \subset \mathbb{C}^{n}$ is an isotropic line, generate $\mathcal{L}_{-}^{\tau}\left(\mathrm{SO}(n, \mathbb{C}), \mathbb{C}^{n}\right)$.

Proof. Let $g \in \mathcal{L}_{-}^{\tau}\left(\mathrm{SO}(n, \mathbb{C}), \mathbb{C}^{n}\right)$ and fix a pole $\alpha \in \mathbb{C} \backslash \mathbb{R}$. As before, we use induction on its degree to remove it. The reality condition implies that $\alpha$ is a pole if and only if $\bar{\alpha}$ is a pole. Thus, in contrast to the case of $\operatorname{CSp}(n, \mathbb{C})$, removing a pole at $\alpha$ simultaneously removes the pole at $\bar{\alpha}$.

We write the Laurant expansion of $g$ in $\frac{\lambda-\alpha}{\lambda-\bar{\alpha}}$ explicitly as

$$
g(\lambda)=\sum_{j=-k}^{\infty}\left(\frac{\lambda-\alpha}{\lambda-\bar{\alpha}}\right)^{j} g_{j},
$$

with $g_{-k} \neq 0$. Since $g$ takes values in $\mathrm{SO}(n, \mathbb{C})$, we have $\langle g(\lambda) v, g(\lambda) w\rangle=\langle v, w\rangle$ for all $v, w \in \mathbb{C}^{n}$ and all $\lambda$ near $\alpha$, where $\langle\cdot, \cdot\rangle$ is the symmetric bilinear form on $\mathbb{C}^{n}$ extending the standard inner product on $\mathbb{R}^{n}$ complex linearly. Expanding this expression in $\lambda$, the terms of lowest order read

$$
\begin{aligned}
\left\langle g_{-k} v, g_{-k} w\right\rangle & =0, \\
\left\langle g_{-k+1} v, g_{-k} w\right\rangle+\left\langle g_{-k} v, g_{-k+1} w\right\rangle & =0 .
\end{aligned}
$$

The first of these equations says that $\operatorname{Im} g_{-k}$ is isotropic. Since no real subspaces are isotropic, we obtain a decomposition

$$
\mathbb{C}^{n}=\operatorname{Im} g_{-k} \oplus\left(\operatorname{Im} g_{-k} \oplus \overline{\operatorname{Im} g_{-k}}\right)^{\perp} \oplus \overline{\operatorname{Im} g_{-k}} .
$$


Now let $L \subset \operatorname{Im} g_{-k}$ be a line and calculate

$$
\begin{aligned}
& \sum_{j=-k-1}^{\infty}\left(\frac{\lambda-\alpha}{\lambda-\bar{\alpha}}\right)^{j} \tilde{g}_{j}:=\tilde{g}(\lambda):=p_{\alpha, L}(\lambda) g(\lambda) \\
& \quad=\left(\frac{\lambda-\alpha}{\lambda-\bar{\alpha}}\right)^{-k-1} \pi_{\bar{L}} g_{-k}+\left(\frac{\lambda-\alpha}{\lambda-\bar{\alpha}}\right)^{-k}\left(\pi_{\bar{L}} g_{-k+1}+\pi_{(L \oplus \bar{L})^{\perp}} g_{-k}\right)+\ldots
\end{aligned}
$$

Since $\operatorname{Im} g_{-k}$ is isotropic we have

$$
\operatorname{ker} \pi_{\bar{L}}=L^{\perp} \supset \operatorname{Im} g_{-k}^{\perp} \supset \operatorname{Im} g_{-k} ;
$$

hence $\tilde{g}_{-k-1}$ vanishes.

It remains to show that the rank $\tilde{g}_{-k}$ is smaller than the rank of $g_{-k}$. For this we compute kernels. Let $v \in \operatorname{ker} g_{-k}$. Then, by (5.4), we have $\left\langle g_{-k+1} v, g_{-k} w\right\rangle=0$ for all $w \in \mathbb{C}^{n}$. Thus $g_{-k+1}\left(\operatorname{ker} g_{-k}\right) \perp \operatorname{Im} g_{-k} \supset L$. In particular we have ker $g_{-k} \subset$ ker $\tilde{g}_{-k}$. To see that this is a proper inclusion we observe that there exists some $v \in \mathbb{C}^{n}$ such that $g_{-k} v \in L \backslash\{0\}$. Applying (5.4) with $w=v$ yields

$$
\left\langle g_{-k+1} v, g_{-k} v\right\rangle=0 \text {. }
$$

Thus $g_{-k+1} v \perp L$ and so $v \in \operatorname{ker} \tilde{g}_{-k}$. We have thus found a vector

$$
v \in \operatorname{ker} \tilde{g}_{-k} \backslash \operatorname{ker} g_{-k} \text {. }
$$

Consequently $\operatorname{rank} \tilde{g}_{-k}<\operatorname{rank} g_{-k}$ and $\tilde{g}$ has a pole of lower degree at $\alpha$.

5.2. Dressing and permutability. The following theorem is well known; see e.g. [2. We include a proof for completeness.

Theorem 5.2. Let $h \in \mathcal{L}(\mathrm{SO}(n, \mathbb{C}))$ be holomorphic at $\alpha \in \mathbb{C} \backslash \mathbb{R}$. If we define $L^{\prime}=h(\alpha)^{-1} L$, then

$$
p_{\alpha, L} h p_{\alpha, L^{\prime}}^{-1}
$$

is holomorphic at $\alpha$.

Proof. Let $h(\lambda)=\sum_{i}\left(\frac{\lambda-\alpha}{\lambda-\bar{\alpha}}\right)^{i} h_{i}$. Multiplying out (5.5), the coefficients of $\left(\frac{\lambda-\alpha}{\lambda-\bar{\alpha}}\right)^{-2}$ and $\left(\frac{\lambda-\alpha}{\lambda-\bar{\alpha}}\right)^{-1}$ are

$$
\begin{aligned}
& \pi_{\bar{L}} h_{0} \pi_{L^{\prime}}, \\
& \pi_{\bar{L}} h_{0} \pi_{\left(L^{\prime} \oplus \overline{L^{\prime}}\right)^{\perp}}+\pi_{(L \oplus \bar{L})^{\perp}} h_{0} \pi_{L^{\prime}}+\pi_{\bar{L}} h_{1} \pi_{L^{\prime}} .
\end{aligned}
$$

Since the image of $h_{0} \pi_{L}^{\prime}$ is $L$, the term (5.6) and the second summand of (5.7) vanish. The first summand of (5.7) is zero because of

$$
\left\langle h_{0}\left(L^{\prime} \oplus \overline{L^{\prime}}\right)^{\perp}, L\right\rangle=\left\langle h_{0}\left(L^{\prime} \oplus \overline{L^{\prime}}\right)^{\perp}, h_{0} L^{\prime}\right\rangle=\left\langle\left(L^{\prime} \oplus \overline{L^{\prime}}\right)^{\perp}, L^{\prime}\right\rangle=0 .
$$

For the vanishing of the third note that $h_{1} h_{0}^{-1} \in \mathfrak{s o}(n, \mathbb{C})$. Then,

$$
\left\langle h_{1} L^{\prime}, L\right\rangle=\left\langle\left(h_{1} h_{0}^{-1}\right) L, L\right\rangle=0 .
$$

It is now is possible to evaluate the loop (5.5) at $\alpha$. The determinant of the loop is 1 at $\alpha$, so since $\mathrm{SO}(n, \mathbb{C})$ is closed in $\operatorname{GL}(n, \mathbb{C})$, the loop is holomorphic at $\alpha$.

Corollary 5.3. Suppose that $p_{\alpha, L}$ and $p_{\beta, M}$ are simple factors such that $\alpha \neq \beta, \bar{\beta}$. Then

$$
p_{\beta, p_{\alpha, L}(\beta) M} p_{\alpha, L}=p_{\alpha, p_{\beta, M}(\alpha) L} p_{\beta, M} .
$$


5.3. Generators for the twisted loop groups. Let $\sigma$ be a holomorphic involution of $\mathrm{SO}(n, \mathbb{C})$ satisfying $\tau \sigma=\sigma \tau$, thereby giving rise to a symmetric space $\mathrm{SO}(n) / K$, where $K$ is the fixed point set of $\sigma$ in $\mathrm{SO}(n)$. Recall that we say a loop $g$ is twisted if

$$
\sigma(g(-\lambda))=g(\lambda) .
$$

Note that for any such $\sigma$, there exists $s \in \mathrm{O}(n)$ such that $\sigma(A)=s A s^{-1}$. In the case of the Grassmannians, $s$ is given by

$$
s=\left(\begin{array}{cc}
I_{k} & 0 \\
0 & -I_{n-k}
\end{array}\right),
$$

for some $k$, and for $\mathrm{SO}(2 m) / \mathrm{U}(m)$,

$$
s=\left(\begin{array}{cc}
0 & I_{m} \\
-I_{m} & 0
\end{array}\right) .
$$

In either case $s \in \mathrm{O}(n)$ sends isotropic lines in $\mathbb{C}^{n}$ to isotropic lines.

Lemma 5.4. If $\alpha \notin i \mathbb{R}$ and $p_{\alpha, L}$ is a simple element, then the product

$$
q_{\alpha, L}(\lambda):=p_{-\alpha, p_{\alpha, L}(-\alpha) s L} p_{\alpha, L}=p_{\alpha, p_{-\alpha, s L}(\alpha) L} p_{-\alpha, s L}
$$

satisfies the twisting condition.

The proof follows immediately from the permutability proposition.

Theorem 5.5. The elements

$$
\begin{gathered}
p_{\alpha, L}(\lambda)=\left(\frac{\lambda-\bar{\alpha}}{\lambda-\alpha}\right) \pi_{\bar{L}}+\pi_{(L \oplus \bar{L})^{\perp}}+\left(\frac{\lambda-\alpha}{\lambda-\bar{\alpha}}\right) \pi_{L}, \quad \text { where } s L=\bar{L} \text {, and } \alpha \in i \mathbb{R}, \\
\text { and } q_{\alpha, L}(\lambda), \quad \text { where } \alpha \in \mathbb{C} \backslash(\mathbb{R} \cup i \mathbb{R}), \text { and } L \subset \mathbb{C}^{n} \text { isotropic, }
\end{gathered}
$$

generate the twisted loop group $\mathcal{L}_{-}^{\tau, \sigma}\left(\mathrm{SO}(n, \mathbb{C}), \mathbb{C}^{n}\right)$.

These loops are precisely those defined as simple elements in [1 and simple factors in 2 .

Proof. Let $g \in \mathcal{L}_{-}^{\tau, \sigma}\left(\mathrm{SO}(n, \mathbb{C}), \mathbb{C}^{n}\right)$. We proceed by induction on degree, considering the cases of purely imaginary poles and nonimaginary poles separately. First assume that $\alpha$ is a nonimaginary pole of $g$. Observe that then, $\bar{\alpha},-\alpha$, and $-\bar{\alpha}$ are also poles of $g$. If we manage to remove the pole at $\alpha$ by successively multiplying with loops satisfying the reality and twisting conditions, we automatically remove these other poles as well. Applying Theorem [5.1. we see that there exists an isotropic line $L$ such that $p_{\alpha, L} g$ has lower total degree at $\alpha$. Then, $q_{\alpha, L} g$ does so, too, and also satisfies the twisting condition. Continuing in the same way, we are able to remove the pole at $\alpha$.

Suppose that $\alpha=i r$ is a purely imaginary pole of $g \in \mathcal{L}_{-}^{\tau, \sigma}\left(\mathrm{SO}(n, \mathbb{C}), \mathbb{C}^{n}\right)$ of degree $k$, and expand $g$ as before.

The twisting and reality conditions

$$
\overline{g(\bar{\lambda})}=g(\lambda)=\sigma g(-\lambda)
$$

imply

$$
\sum_{j=-k}^{\infty}\left(\frac{\lambda+i r}{\lambda-i r}\right)^{j} \overline{g_{j}}=\sum_{j=-k}^{\infty}\left(\frac{\lambda+i r}{\lambda-i r}\right)^{j} \sigma g_{j}
$$


hence $\sigma g_{j}=\overline{g_{j}}$ for all $j$. In particular $\sigma g_{-k}=\overline{g_{-k}}$, from which we see that $\overline{\operatorname{Im} g_{-k}}=\operatorname{Im} \overline{g_{-k}}=\operatorname{Im} \sigma g_{-k}=s\left(\operatorname{Im} g_{-k}\right)$. Therefore, $s$ composed with conjugation is an involutive endomorphism of $\operatorname{Im} g_{-k}$, and we may choose a fixed line $L$; i.e., $s \bar{L}=L \subset \operatorname{Im} g_{-k}$.

By the proof of Theorem 5.1, the map $p_{i r, L} g$ has a pole of lower total degree at ir than $g$. This loop clearly satisfies the reality and twisting conditions and is thus in $\mathcal{L}_{-}^{\tau, \sigma}\left(\mathrm{SO}(n, \mathbb{C}), \mathbb{C}^{n}\right)$. By induction we may entirely factor out the poles on $i \mathbb{R}^{\times}$.

As before we conclude from Liouville's theorem that we have reduced the given loop to the identity using simple factors.

\section{6. $\mathbb{G}_{2}$}

6.1. The linear algebra of $\mathbb{G}_{2}$ and its fundamental representation. Let $\mathbb{O}$ denote the octonions, the unique real 8-dimensional division algebra, equipped with the natural metric $\langle x, y\rangle=\operatorname{Re}(x \cdot \bar{y})=\frac{1}{2}(x \cdot \bar{y}+y \cdot \bar{x})$. The compact simple Lie group $\mathrm{G}_{2}$ is known to be the automorphism group of $\mathbb{O}$. Since the metric is defined via the multiplication, we get $\mathrm{G}_{2} \subset \mathrm{SO}(\mathbb{O})$. The subspace $\mathbb{R} \cdot 1 \subset \mathbb{O}$ is fixed by $\mathrm{G}_{2}$, so if we identify $\operatorname{Im}(\mathbb{O})=\mathbb{R}^{7}$, we obtain the fundamental representation $\mathrm{G}_{2} \subset \mathrm{SO}(7)$.

Let $1, e_{1}, \ldots, e_{7}$ be the standard orthonormal basis of $\mathbb{R}^{8} \cong \mathbb{O}$. The octonionic multiplication table is displayed in Appendix A.

\section{Definition 6.1.}

- A 3-plane $A \subset \mathbb{R}^{7}$ is associative if it generates an associative subalgebra of $\mathbb{0}$.

- A 4-plane $C \subset \mathbb{R}^{7}$ is coassociative if $C^{\perp}$ is associative.

Remark 6.2. In the literature the title (co)associative also implies the choice of a particular orientation which makes the plane calibrated 9]. We do not need the calibrations or the orientations and so use a slightly weaker definition.

Our convention was chosen so that $e_{1} \wedge e_{2} \wedge e_{3} \wedge e_{4}$ is coassociative. As they will be heavily relied upon here, we record the following well-known facts concerning the action of $\mathrm{G}_{2}$ on $\mathbb{R}^{7}$.

\section{Lemma 6.3.}

(1) Each 2-plane $E \subset \mathbb{R}^{7}$ is contained in a unique associative 3-plane $E_{+}$.

(2) $\mathrm{G}_{2}$ acts transitively on the Grassmannian of oriented 2-planes $E \subset \mathbb{R}^{7}$ with stabilizer $\mathrm{U}(2)$ acting irreducibly on $\left(E_{+}\right)^{\perp}$.

In $\mathrm{SO}(4)=\operatorname{Stab}\left(e_{1} \wedge e_{2} \wedge e_{3} \wedge e_{4}\right)$ we can choose the torus $T$ whose Lie algebra is the span of

$$
H_{1}=\left(\begin{array}{ccccccc}
0 & -1 & 0 & 0 & 0 & 0 & 0 \\
1 & 0 & 0 & 0 & 0 & 0 & 0 \\
0 & 0 & 0 & 1 & 0 & 0 & 0 \\
0 & 0 & -1 & 0 & 0 & 0 & 0 \\
0 & 0 & 0 & 0 & 0 & 0 & 0 \\
0 & 0 & 0 & 0 & 0 & 0 & 0 \\
0 & 0 & 0 & 0 & 0 & 0 & 0
\end{array}\right) \text { and } H_{2}=\left(\begin{array}{ccccccc}
0 & -1 & 0 & 0 & 0 & 0 & 0 \\
1 & 0 & 0 & 0 & 0 & 0 & 0 \\
0 & 0 & 0 & -1 & 0 & 0 & 0 \\
0 & 0 & 1 & 0 & 0 & 0 & 0 \\
0 & 0 & 0 & 0 & 0 & 0 & 0 \\
0 & 0 & 0 & 0 & 0 & 0 & -2 \\
0 & 0 & 0 & 0 & 0 & 2 & 0
\end{array}\right) .
$$

Let $\mathbb{O}_{\mathbb{C}}=\mathbb{O} \otimes \mathbb{C}$; then $\mathbb{G}_{2}:=\mathrm{G}_{2}^{\mathbb{C}} \subset \mathrm{SO}(7, \mathbb{C})$ is the automorphism group of $\mathbb{O}_{\mathbb{C}}$. From now on, if $x, y \in \mathbb{C}^{7}=\operatorname{Im}(\mathbb{O}) \otimes \mathbb{C}$, the product $x \cdot y \in \mathbb{C}^{7}$ will denote the octonionic-imaginary part of the product of $x$ and $y$. 
With respect to the torus $T$, the weights of the $\mathbb{G}_{2}$-representation on $\mathbb{C}^{7}$ are $\mu_{0}=0, \pm \mu_{1}, \pm \mu_{2}, \pm \mu_{3}$, where

$$
\begin{array}{r}
\mu_{1}\left(H_{1}\right)=-i, \quad \mu_{1}\left(H_{2}\right)=-i, \\
\mu_{2}\left(H_{1}\right)=-i, \quad \mu_{2}\left(H_{2}\right)=i, \\
\mu_{3}\left(H_{1}\right)=0, \quad \mu_{3}\left(H_{2}\right)=2 i
\end{array}
$$

and the weight spaces $L_{i}$ of $\mu_{i}$ are given by $L_{1}=\mathbb{C} \cdot\left\{e_{1}+i e_{2}\right\}, L_{2}=\mathbb{C} \cdot\left\{e_{3}-i e_{4}\right\}$, $L_{3}=\mathbb{C} \cdot\left\{e_{6}-i e_{7}\right\}$, and $L_{0}=\mathbb{C} \cdot\left\{e_{5}\right\}$. The product $L_{i} \cdot L_{j}$ is the weight space for $\mu_{i}+\mu_{j}$, if this is a weight, and zero otherwise. For example, $L_{1} \cdot L_{3}=L_{2}$.

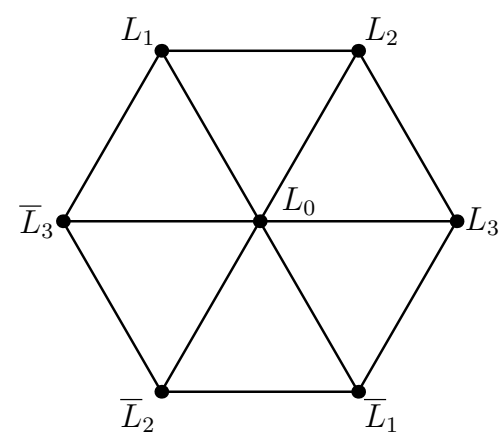

FIGURE 1. Weight diagram for the fundamental representation of $\mathbb{G}_{2}$.

Definition 6.4. An isotropic 2-plane $\mathcal{C} \subset \mathbb{C}^{7}$ satisfying $\mathcal{C} \cdot \mathcal{C}=0$ is complex coassociative.

Either from the weight diagram or the multiplication table in the appendix, one calculates that $L_{1} \cdot L_{2}=0$, i.e. $L_{1} \oplus L_{2}$ is complex coassociative. Any complex coassociative plane $\mathcal{C}$ is isotropic by definition and thus induces an orthogonal decomposition

$$
\mathbb{C}^{7}=\mathcal{C} \oplus \mathcal{A} \oplus \overline{\mathcal{C}},
$$

where $\mathcal{A}=(\mathcal{C} \oplus \overline{\mathcal{C}})^{\perp}$ is called complex associative.

Lemma 6.5. Let $L \subset \mathbb{C}^{7}$ be an isotropic line.

(1) There is a unique isotropic 2-plane $\mathcal{B} \subset(L \oplus \bar{L})^{\perp}$ such that any line in $\mathcal{B}$ multiplies with $L$ to be zero. Conversely, for any complex coassociative $\mathcal{C} \supset L$ we can choose a $K \in \mathcal{B}$ such that $\mathcal{C}=L \oplus K$.

(2) If $K$ is any other line, then $L \cdot K=0$ implies that $K$ is isotropic, and therefore $L \oplus K$ is complex coassociative.

Proof. By part two of Lemma 6.3, we can assume without loss of generality that $L=L_{1}$. Then $(L \oplus \bar{L})^{\perp}=L_{2} \oplus \overline{L_{2}} \oplus L_{3} \oplus \overline{L_{3}} \oplus L_{0}$. A look at the weight diagram shows that $\mathcal{B}=L_{2} \oplus \overline{L_{3}}$, which shows part one. Part two follows from the fact that $K$ has to lie in the isotropic 3-plane $L \oplus \mathcal{B}$.

Proposition 6.6. The compact real group $\mathrm{G}_{2}$ acts transitively on the space of complex coassociative planes. 
Proof. Let $\mathcal{C}=L \oplus K$ be a decomposition of a complex coassociative plane into lines such that $L \perp \bar{K}$. By part two of Lemma 6.3. there is $g \in \mathrm{G}_{2}$ with $g L=L_{1}$. By part one of Lemma 6.5. we know that $g K \subset L_{2} \oplus \overline{L_{3}}$. Then by part two of Lemma 6.3, there exists $h \in \mathrm{G}_{2}$ with $h L_{1}=L_{1}$ and $h g K=L_{2}$. We have shown that any complex coassociative plane can be mapped to $L_{1} \oplus L_{2}$.

Corollary 6.7. The intersection $C=(\mathcal{C} \oplus \overline{\mathcal{C}}) \cap \mathbb{R}^{7}$ is a coassociative 4-plane and $\mathcal{A}$ is the complexification of an associative 3 -plane $A \subset \mathbb{R}^{7}$.

Corollary 6.8. If $\mathcal{C}$ and $\mathcal{D}$ are complex coassociative and $\mathcal{C} \cap \mathcal{D} \neq 0$, then they are orthogonal.

Lemma 6.9. The octonionic multiplication table for the decomposition (6.1) is given by

\begin{tabular}{c||c|c|c} 
& $\mathcal{C}$ & $\mathcal{A}$ & $\overline{\mathcal{C}}$ \\
\hline \hline $\mathcal{C}$ & 0 & $\mathcal{C}$ & $\mathcal{A}$ \\
\hline $\mathcal{A}$ & $\mathcal{C}$ & $\mathcal{A}$ & $\overline{\mathcal{C}}$ \\
\hline$\overline{\mathcal{C}}$ & $\mathcal{A}$ & $\overline{\mathcal{C}}$ & 0
\end{tabular}

Proof. Because $\mathrm{G}_{2}$ acts transitively on complex coassociative planes, one only has to check this using the multiplication table from Appendix $\mathrm{A}$ for the standard complex coassociative plane $L_{1} \oplus L_{2}$.

6.2. Generating $\mathcal{L}\left(\mathbb{G}, \mathbb{C}^{7}\right)$. As before, let $\mathbb{G}_{2} \subset \mathrm{SO}(7, \mathbb{C})$ be the complexification of $\mathrm{G}_{2} \subset \mathrm{SO}(7)$. Conjugation on $\mathrm{SO}(7, \mathbb{C})$ restricts to an involution $\tau$ on $\mathbb{G}_{2}$ whose fixed point set is $\mathrm{G}_{2}$. First we need to describe the simple elements.

We use the notation of Section 6.1 The element $i H_{1}$ is $\rho$-integral. By Proposition 3.5. the simple element $\exp \left(\ln \left(\frac{\lambda-\alpha}{\lambda-\bar{\alpha}}\right) i H_{1}\right)$ acts on $\mathbb{C}^{7}$ as

$$
\left(\frac{\lambda-\bar{\alpha}}{\lambda-\alpha}\right) \pi_{\overline{\mathcal{C}_{0}}}+\pi_{\mathcal{A}_{0}}+\left(\frac{\lambda-\alpha}{\lambda-\bar{\alpha}}\right) \pi_{\mathcal{C}_{0}}
$$

where $\mathcal{C}_{0}=L_{1} \oplus L_{2}$ and $\overline{\mathcal{C}_{0}}=\overline{L_{1}} \oplus \overline{L_{2}}$ are complex coassociative planes and $\mathcal{A}_{0}=L_{3} \oplus L_{0} \oplus \overline{L_{3}}$ is a complex associative 3-plane. Conversely, given any element of the form

$$
p_{\alpha, \mathcal{C}}(\lambda):=\left(\frac{\lambda-\bar{\alpha}}{\lambda-\alpha}\right) \pi_{\overline{\mathcal{C}}}+\pi_{\mathcal{A}}+\left(\frac{\lambda-\alpha}{\lambda-\bar{\alpha}}\right) \pi_{\mathcal{C}}
$$

where $\mathcal{C}$ is an arbitrary complex coassociative plane and $\mathcal{A}=(\mathcal{C} \oplus \overline{\mathcal{C}})^{\perp}$, Proposition 6.6 implies that it is conjugate to the simple element defined by $H_{1}$, so it is a simple element itself.

Theorem 6.10. The simple elements $p_{\alpha, \mathcal{C}}$ generate $\mathcal{L}_{-}^{\tau}\left(\mathbb{G}_{2}, \mathbb{C}^{7}\right)$.

Proof. Let $\alpha$ be a pole of $g$ and expand $g$ as before. Because $g$ is a map into $\mathrm{SO}(7, \mathbb{C})$, equations (5.3) and (5.4) may be used here:

$$
\begin{aligned}
\left\langle g_{-k}(v), g_{-k}(w)\right\rangle & =0, \\
\left\langle g_{-k}(v), g_{-k+1}(w)\right\rangle+\left\langle g_{-k+1}(v), g_{-k}(w)\right\rangle & =0 .
\end{aligned}
$$


For all $v, w \in \mathbb{C}^{7}$, we have $g(\lambda)(v \cdot w)=g(\lambda)(v) \cdot g(\lambda)(w)$. Expanding this equation and comparing the two coefficients of lowest order, we obtain

$$
\begin{aligned}
g_{-k}(v) \cdot g_{-k}(w) & =0, \\
g_{-k}(v) \cdot g_{-k+1}(w)+g_{-k+1}(v) \cdot g_{-k}(w) & = \begin{cases}0, & k \geq 2, \\
g_{-1}(v \cdot w), & k=1 .\end{cases}
\end{aligned}
$$

It follows from equations (6.2) and (6.4) that $\operatorname{Im}\left(g_{-k}\right)$ is either an isotropic line or a complex coassociative plane; thus $\operatorname{rank}\left(g_{-k}\right) \leq 2$.

Assume $k \geq 2$; the case of $k=1$ will be dealt with later. Choose $L \subset \operatorname{Im}\left(g_{-k}\right)$ and $v$ such that $0 \neq g_{-k}(v) \in L$. By Lemma 6.5 there exists a line $M \subset(L \oplus$ $\bar{L})^{\perp}$ such that $\mathcal{C}=L \oplus M$ is complex coassociative and $g_{-k+1}(v) \perp M$. Note that $\mathcal{C}$ is perpendicular to $\operatorname{Im}\left(g_{-k}\right)$, since $\operatorname{Im}\left(g_{-k}\right)$ is either equal to $L \subset \mathcal{C}$ or a complex coassociative plane intersecting $\mathcal{C}$ nontrivially, whereupon Corollary 6.8 is applicable.

We claim that $p_{\alpha, \mathcal{c}} g$ has a pole of strictly lower total degree at zero. Multiplying out, we get

$$
\begin{aligned}
p_{\alpha, \mathcal{C}}(\lambda) g(\lambda) & =\left(\frac{\lambda-\alpha}{\lambda-\bar{\alpha}}\right)^{-k-1} \pi_{\overline{\mathcal{C}}} g_{-k}+\left(\frac{\lambda-\alpha}{\lambda-\bar{\alpha}}\right)^{-k}\left(\pi_{\left.\overline{\mathcal{C}} g_{-k+1}+\pi_{\mathcal{A}} g_{-k}\right)+\ldots}+\sum_{j=-k-1}^{\infty}\left(\frac{\lambda-\alpha}{\lambda-\bar{\alpha}}\right)^{j} \tilde{g}_{j} .\right.
\end{aligned}
$$

Since $\mathcal{C}$ and $\operatorname{Im}\left(g_{-k}\right)$ are orthogonal, $\tilde{g}_{-k-1}=\pi_{\overline{\mathcal{C}}} g_{-k}=0$. We need to show that the rank of $\tilde{g}_{-k}$ is smaller than the rank of $g_{-k}$. For this, we compare the kernels.

If $w \in \operatorname{ker}\left(g_{-k}\right)$, then equation (6.5) gives

$$
g_{-k}(v) \cdot g_{-k+1}(w)=0
$$

which implies that $g_{-k+1}(w) \cdot L=0$; i.e. $g_{-k+1}(w)$ is either contained in $L$, which immediately implies that it is perpendicular to $\mathcal{C}$, or, together with $L$ spans a complex coassociative plane, whereupon it is perpendicular to $\mathcal{C}$ by Corollary 6.8 . It follows that $\tilde{g}_{-k}(w)=0$, i.e. $\operatorname{ker} g_{-k} \subset \operatorname{ker} \tilde{g}_{-k}$.

To show that we have a strict inclusion, we show that $\tilde{g}_{-k}(v)=0$. We have $\pi_{\mathcal{A}} g_{-k}(v)=0$, so we need to show that $g_{-k+1}(v)$ is perpendicular to $\mathcal{C}$. By definition, $g_{-k+1}(v)$ is perpendicular to $M$. Equation (6.3) with $v=w$ implies that it is perpendicular to $L$, so it is perpendicular to $\mathcal{C}$.

Now assume $k=1$. From equation (6.5) we find that $\operatorname{ker}\left(g_{-1}\right)$ is a multiplicatively closed subspace of $\mathbb{C}^{7}$. Since such subspaces can at most be five-dimensional, it follows that $\operatorname{rank}\left(g_{-1}\right)=2$. The loop $g$ may also be regarded as a loop in $\operatorname{SO}(7, \mathbb{C})$, so applying the Generating Theorem, Theorem 5.1, for $\mathcal{L}_{-}^{\tau}\left(\mathrm{SO}(7, \mathbb{C}), \mathbb{C}^{7}\right)$, we see that there exist two isotropic lines $L, K \subset \mathbb{C}^{7}$ such that

$$
g=p_{\alpha, L} p_{\alpha, K} \tilde{g},
$$

where $\tilde{g}$ is holomorphic at $\alpha$, and $p_{\alpha, L}$ and $p_{\alpha, K}$ are given by (5.1). Multiplying out (6.7), the coefficient of order -2 is $\pi_{\bar{L}} \pi_{\bar{K}} \tilde{g}(\alpha)$. Because $g$ has a pole of order 1 at $\alpha(k=1)$ and $\tilde{g}(\alpha)$ is invertible, it follows that $K \subset \bar{L}^{\perp}$. The coefficient of order -1 is

$$
\left(\pi_{L} \pi_{(K \oplus \bar{K})^{\perp}}+\pi_{(L \oplus \bar{L})^{\perp}} \pi_{K}\right) \tilde{g}(\alpha)
$$


which has the two-dimensional image $\mathcal{C}:=L \oplus \pi_{(L \oplus \bar{L})^{\perp}} K$. The plane $\mathcal{C}$ is complex coassociative by (6.4). By Proposition 6.6. without loss of generality we can assume that $L=L_{1}$ and $\pi_{(L \oplus \bar{L})^{\perp}} K=L_{2}$ in the weight diagram. We have that $K \subset$ $\pi_{(L \oplus \bar{L})^{\perp}} K \oplus \bar{L}=L_{2} \oplus \overline{L_{1}}$. Note that $p_{\alpha, L} p_{\alpha, K}$ is not necessarily equal to $p_{\alpha, \mathcal{C}}$; this is only the case if $K$ is contained in $(L \oplus \bar{L})^{\perp}$, i.e. $K=L_{2}$. Having a look at the weight diagram, we see that the isotropic line $R=L_{3} \subset \mathcal{A}$ satisfies $L \cdot \bar{R}=0$ and $K \cdot R=0$ so that both $p_{\alpha, L} p_{\alpha, \bar{R}}$ and $p_{\alpha, R} p_{\alpha, K}$ are simple factors. Thus

$$
g=p_{\alpha, L} p_{\alpha, K} \tilde{g}=\left(p_{\alpha, L} p_{\alpha, \bar{R}}\right)\left(p_{\alpha, R} p_{\alpha, K}\right) \tilde{g}
$$

has been factored.

The proof of the $k=1$ case of the theorem implies the following proposition, which will be used in the discussion of the dressing transformation and permutability.

Proposition 6.11. A pair of isotropic lines $L, K$ satisfies

$$
\begin{aligned}
K & \subset \bar{L}^{\perp} \text { and } \\
\mathcal{C} & =L \oplus \pi_{(L \oplus \bar{L})^{\perp}} K \text { is complex coassociative }
\end{aligned}
$$

if and only $p_{\alpha, L} p_{\alpha, K} \in \mathcal{L}_{-}^{\tau}\left(\mathbb{G}_{2}, \mathbb{C}^{7}\right)$. In this case the product only has simple poles at $\alpha$ and $\bar{\alpha}$. In fact, any $g \in \mathcal{L}_{-}^{\tau}\left(\mathbb{G}_{2}, \mathbb{C}^{7}\right)$ whose only poles are simple ones at $\alpha$ and $\bar{\alpha}$ with $\alpha \in \mathbb{C} \backslash \mathbb{R}$ can be factored as $g=p_{\alpha, L} p_{\alpha, K}$. Furthermore, either

(1) $K \subset(L \oplus \bar{L})^{\perp}$, in which case $\mathcal{C}=L \oplus K$ and

$$
g=p_{\alpha, \mathcal{C}}
$$

or

(2) $K \not \subset(L \oplus \bar{L})^{\perp}$ and then there exists an isotropic line $R$ such that $\mathcal{C}_{1}=L \oplus R$ and $\mathcal{C}_{2}=\bar{R} \oplus K$ are complex coassociative and

$$
g=p_{\alpha, \mathcal{C}_{1}} p_{\alpha, \mathcal{C}_{2}} .
$$

6.3. Dressing and permutability. Any rational loop whose only poles are simple ones at $\alpha$ and $\bar{\alpha}$ with $\alpha \in \mathbb{C} \backslash \mathbb{R}$ is, by Proposition 6.11 of the form $p_{\alpha, L, K}=p_{\alpha, L} p_{\alpha, K}$ for two isotropic lines $L$ and $K$ for which (6.10) holds. The notation $p_{\alpha, L, K}$ will only be used for lines $L$ and $K$ satisfying these conditions. Dressing of positive loops with $p_{\alpha, L, K}$ is performed by using the dressing action in $\mathrm{SO}(7, \mathbb{C})$ for each of the $\mathrm{SO}(7, \mathbb{C})$-factors. In order to prove a permutability formula we also need to use a dressing-type action of the rational loop group on itself.

Let $h \in \mathcal{L}_{-}^{\tau}\left(\mathbb{G}_{2}, \mathbb{C}^{7}\right)$ be holomorphic at $\alpha$ (and thus $\left.\bar{\alpha}\right)$. Define

$$
\begin{aligned}
K^{\prime} & =h(\alpha)^{-1} K, \\
p_{\alpha, K} * h & =p_{\alpha, K} h p_{\alpha, K^{\prime}}^{-1}
\end{aligned}
$$

From the dressing theorem for $\mathrm{SO}(n, \mathbb{C})$ we know that $p_{\alpha, K} * h$ is holomorphic at $\alpha$, allowing us to define

$$
L^{\prime}=\left(p_{\alpha, K} * h\right)(\alpha)^{-1} L
$$

Proposition 6.12. The pair of lines $L^{\prime}, K^{\prime}$ satisfies (6.10). Therefore, $p_{\alpha, L^{\prime}, K^{\prime}} \in$ $\mathcal{L}_{-}^{\tau}\left(\mathbb{G}_{2}, \mathbb{C}^{7}\right)$ has simple poles at $\alpha$ and $\bar{\alpha}$. Furthermore,

$$
p_{\alpha, L, K} h p_{\alpha, L^{\prime}, K^{\prime}}^{-1}
$$

is in $\mathcal{L}_{-}^{\tau}\left(\mathbb{G}_{2}, \mathbb{C}^{7}\right)$ and holomorphic at $\alpha$ and $\bar{\alpha}$. 
Proof. That the resulting element is holomorphic follows from applying Theorem 5.2 twice. We will now show that it is a loop in $\mathbb{G}_{2}$. By the generating theorem for $\mathcal{L}_{-}^{\tau}\left(\mathbb{G}_{2}, \mathbb{C}^{7}\right)$,

$$
p_{\alpha, L, K} h=\hat{h} p_{\alpha, M, N},
$$

where $\hat{h}$ is holomorphic at $\alpha$ and $\bar{\alpha}$ and $p_{\alpha, M, N}$ is a loop in $\mathbb{G}_{2}$. That we do not have a more complicated product of simple elements having poles at $\alpha$ and $\bar{\alpha}$ is due to the fact that the left hand side has only simple poles there. Therefore

$$
p_{\alpha, L, K} h p_{\alpha, L^{\prime}, K^{\prime}}^{-1} \hat{h}^{-1}=\hat{h} p_{\alpha, M, N} p_{\alpha, L^{\prime}, K^{\prime}}^{-1} \hat{h}^{-1} .
$$

The left hand side is holomorphic at $\alpha$, so $p_{\alpha, M, N} p_{\alpha, L^{\prime}, K^{\prime}}^{-1}$ is constant. Since all factors were normalized to be the identity at infinity, $p_{\alpha, L^{\prime}, K^{\prime}}=p_{\alpha, M, N}$ and is thus in $\mathcal{L}_{-}^{\tau}\left(\mathbb{G}_{2}, \mathbb{C}^{7}\right)$.

Corollary 6.13 (Permutability). Let $L, K$ and $M, N$ be pairs of lines satisfying (6.10). Then, for all $\alpha, \beta \in \mathbb{C} \backslash \mathbb{R}$ with $\alpha \neq \beta, \bar{\beta}$, we have

$$
p_{\alpha, L^{\prime}, K^{\prime}} p_{\beta, M, N}=p_{\beta, M^{\prime}, N^{\prime}} p_{\alpha, L, K},
$$

where

$$
\begin{aligned}
K^{\prime} & =p_{\beta, M, N}(\alpha) K, \\
L^{\prime} & =\left(p_{\alpha, K^{\prime}} * p_{\beta, M, N}\right)(\alpha) L, \\
N^{\prime} & =p_{\alpha, L, K}(\beta) N, \\
M^{\prime} & =\left(p_{\beta, N^{\prime}} * p_{\alpha, L, K}\right)(\beta) M .
\end{aligned}
$$

6.4. Generators for the twisted loop group. There is a unique irreducible compact Riemannian symmetric space whose isometry group is $\mathrm{G}_{2}$. It is defined by the automorphism $\sigma(g)=s g s^{-1}$, where

$$
s=\left(\begin{array}{cc}
-I_{4} & 0 \\
0 & I_{3}
\end{array}\right) \in \mathrm{G}_{2}
$$

and $\mathrm{G}_{2}^{\sigma}=\mathrm{SO}(4)$. In this section we introduce generators for the twisted loop group $\mathcal{L}_{-}^{\tau, \sigma}\left(\mathbb{G}_{2}, \mathbb{C}^{7}\right)$.

Lemma 6.14. For $p_{\alpha, L, K}$ as above, the loop

$$
q_{\alpha, L, K}=p_{-\alpha,(s L)^{\prime},(s K)^{\prime}} p_{\alpha, L, K}
$$

satisfies the twisting condition $\sigma\left(q_{\alpha, L, K}(-\lambda)\right)=q_{\alpha, L, K}(\lambda)$, where

$$
\begin{aligned}
(s K)^{\prime} & =p_{\alpha, L, K}(-\alpha) s K, \\
(s L)^{\prime} & =\left(p_{-\alpha,(s K)^{\prime}} * p_{\alpha, L, K}\right)(-\alpha) s L .
\end{aligned}
$$

Proof. Corollary 6.13 yields

$$
q_{\alpha, L, K}=p_{-\alpha,(s L)^{\prime},(s K)^{\prime}} p_{\alpha, L, K}=p_{\alpha, L^{\prime}, K^{\prime}} p_{-\alpha, s L, s K},
$$

where

$$
\begin{aligned}
K^{\prime} & =p_{-\alpha, s L, s K}(\alpha) K, \\
L^{\prime} & =\left(p_{\alpha, K^{\prime}} * p_{-\alpha, s L, s K}(\alpha) L .\right.
\end{aligned}
$$

Note that

$$
s(s K)^{\prime}=K^{\prime} \text { and } s(s L)^{\prime}=L^{\prime} .
$$


The equations

$$
\sigma\left(p_{\alpha, L, K}(-\lambda)\right)=p_{-\alpha, s L, s K}(\lambda)
$$

and

$$
\sigma\left(p_{-\alpha,(s L)^{\prime},(s K)^{\prime}}(-\lambda)\right)=p_{\alpha, s(s L)^{\prime}, s(s K)^{\prime}}(\lambda)=p_{\alpha, L^{\prime}, K^{\prime}}(\lambda)
$$

combine to

$$
\sigma\left(q_{\alpha, L, K}(-\lambda)\right)=\left(p_{\alpha, L^{\prime}, K^{\prime}} p_{-\alpha, s L, s K}\right)(\lambda) .
$$

This, together with (6.14), gives the desired result.

Theorem 6.15. The elements $p_{\beta, M, N}$, with $\beta \in i \mathbb{R}$ and $s M=\bar{M}$ and $s N=\bar{N}$, and $q_{\alpha, L, K}=p_{-\alpha,(s L)^{\prime},(s K)^{\prime}} p_{\alpha, L, K}$ generate $\mathcal{L}_{-}^{\tau, \sigma}\left(\mathbb{G}_{2}, \mathbb{C}^{7}\right)$.

Remark 6.16. Note that $L$ and $K$ are only assumed to satisfy condition (6.10).

Proof. Let $g \in \mathcal{L}_{-}^{\tau, \sigma}\left(\mathbb{G}_{2}, \mathbb{C}^{7}\right)$. We use the same induction proof that was used for the generating theorem for $\mathrm{SO}(n)$-symmetric spaces. If the order of a pole is greater than one, we may reduce its order using elements of the form $p_{\beta, \mathcal{C}}$ and $q_{\alpha, L, K}$, with $L \oplus K$ being complex coassociative. The only new difficulty occurs for poles of order 1 , so suppose we have applied the induction step until only such poles remain.

We first regard the case of a purely imaginary pole $\beta$ of order 1 . As in the ' $k=1$ 'part of the proof of the generating theorem for $\mathcal{L}_{-}^{\tau}\left(\mathbb{G}_{2}, \mathbb{C}^{7}\right)$, we find isotropic lines $M$ and $N$ such that

$$
g=p_{\beta, M, N} h,
$$

where $h$ is holomorphic at $\beta$, the line $N$ can be written as $N=R+t \bar{M}$ with $R \subset(M \oplus \bar{M})^{\perp}$ and $t \in \mathbb{C}$, and $\mathcal{C}=M \oplus R$ is complex coassociative. Note that there are many choices for $M$ and $N$; later, we will choose a particular line $M$. The loop $p_{\beta, M, N}$ has poles only at $\beta$ and $\bar{\beta}$, and because $\beta \in i \mathbb{R}$, the loop $\sigma\left(p_{\beta, M, N}(-\lambda)\right)$ has the same poles. Then,

$$
\sigma\left(p_{\beta, M, N}(-\lambda)\right) \sigma(h(-\lambda))=\sigma(g(-\lambda))=g(\lambda)=p_{\beta, M, N}(\lambda) h(\lambda)
$$

and therefore

$$
p_{\beta, M, N}(\lambda)^{-1} \sigma\left(p_{\beta, M, N}(-\lambda)\right)=h(\lambda) \sigma(h(-\lambda))^{-1} .
$$

The right hand side is holomorphic at $\beta$ and $\bar{\beta}$, so $p_{\beta, L, K}$ satisfies the twisting condition.

The symmetries $\sigma(g)=g$ and $\bar{\beta}=-\beta$ ensure that we may additionally assume $s M=\bar{M}$. Since $p_{\beta, M, N}$ satisfies the twisting, from this choice of $M$ it follows that $s N=\bar{N}$.

Let $\alpha \in \mathbb{C} \backslash(\mathbb{R} \cup i \mathbb{R})$ be a pole of $g$ of order 1 . By the generating theorem for $\mathcal{L}_{-}^{\tau}\left(\mathbb{G}_{2}, \mathbb{C}^{7}\right)$, we find $L$ and $K$ such that the loop

$$
p_{\alpha, L, K} g
$$

is holomorphic at $\alpha$ and $-\alpha$. Since $p_{-\alpha,(s L)^{\prime},(s K)^{\prime}}$ is holomorphic at $\alpha$ and $\bar{\alpha}$, the loop

$$
q_{\alpha, L, K} g=p_{-\alpha,(s L)^{\prime},(s K)^{\prime}} p_{\alpha, L, K} g
$$

is also holomorphic at $\alpha$ and $\bar{\alpha}$ and satisfies the twisting condition. 


\section{Appendix A. An explicit Description of $\mathrm{G}_{2} \subset \mathrm{SO}(7)$}

Let $(\mathbb{O}$ denote the octonions, the unique real 8-dimensional division algebra, equipped with the natural metric $\langle x, y\rangle=\operatorname{Re}(x \cdot \bar{y})=\frac{1}{2}(x \cdot \bar{y}+y \cdot \bar{x})$. The compact simple Lie group $\mathrm{G}_{2}$ is known to be the automorphism group of $\mathbb{O}$. Since the metric is defined via the multiplication, we get $\mathrm{G}_{2} \subset \mathrm{SO}(\mathbb{O})$. The subspace $\mathbb{R} \cdot 1 \subset \mathbb{O}$ is fixed by $\mathrm{G}_{2}$, so if we identify $\mathbb{R}^{7}=\operatorname{Im}(\mathbb{O})$, we obtain the fundamental representation $\mathrm{G}_{2} \subset \mathrm{SO}(7)$.

Let $1, e_{1}, \ldots, e_{7}$ be the standard orthonormal basis of $\mathbb{R}^{8} \cong \mathbb{O}$. Our convention is that they satisfy the multiplication table:

\begin{tabular}{c||c|c|c|c|c|c|c|c} 
& 1 & $e_{1}$ & $e_{2}$ & $e_{3}$ & $e_{4}$ & $e_{5}$ & $e_{6}$ & $e_{7}$ \\
\hline \hline 1 & 1 & $e_{1}$ & $e_{2}$ & $e_{3}$ & $e_{4}$ & $e_{5}$ & $e_{6}$ & $e_{7}$ \\
\hline$e_{1}$ & $e_{1}$ & -1 & $-e_{5}$ & $-e_{6}$ & $-e_{7}$ & $e_{2}$ & $e_{3}$ & $e_{4}$ \\
\hline$e_{2}$ & $e_{2}$ & $e_{5}$ & -1 & $-e_{7}$ & $e_{6}$ & $-e_{1}$ & $-e_{4}$ & $e_{3}$ \\
\hline$e_{3}$ & $e_{3}$ & $e_{6}$ & $e_{7}$ & -1 & $-e_{5}$ & $e_{4}$ & $-e_{1}$ & $-e_{2}$ \\
\hline$e_{4}$ & $e_{4}$ & $e_{7}$ & $-e_{6}$ & $e_{5}$ & -1 & $-e_{3}$ & $e_{2}$ & $-e_{1}$ \\
\hline$e_{5}$ & $e_{5}$ & $-e_{2}$ & $e_{1}$ & $-e_{4}$ & $e_{3}$ & -1 & $e_{7}$ & $-e_{6}$ \\
\hline$e_{6}$ & $e_{6}$ & $-e_{3}$ & $e_{4}$ & $e_{1}$ & $-e_{2}$ & $-e_{7}$ & -1 & $e_{5}$ \\
\hline$e_{7}$ & $e_{7}$ & $-e_{4}$ & $-e_{3}$ & $e_{2}$ & $e_{1}$ & $e_{6}$ & $-e_{5}$ & -1
\end{tabular}

From the multiplication table one can calculate that a matrix $X \in \mathfrak{s o}(7)$ is in $\mathfrak{g}_{2}$ if and only if it satisfies

$$
\begin{aligned}
& X_{12}+X_{34}-X_{67}=0, \\
& X_{13}+X_{42}-X_{75}=0, \\
& X_{14}+X_{23}-X_{56}=0, \\
& X_{51}+X_{64}-X_{73}=0, \\
& X_{52}+X_{63}+X_{74}=0, \\
& X_{53}-X_{62}+X_{71}=0, \\
& X_{54}-X_{61}-X_{72}=0 .
\end{aligned}
$$

Let $S=\left(\begin{array}{cc}-I_{4} & 0 \\ 0 & I_{3}\end{array}\right)$ and $\sigma(X)=S X S^{-1}$. This induces the Cartan decomposition $\mathfrak{g}_{2}=\mathfrak{s o}(4) \oplus \mathfrak{p}$. Adapting the description of $\mathfrak{g}_{2}$ to this decomposition, we can write an element as

$$
X=\left(\begin{array}{cc}
A & -{ }^{t} B \\
B & \nu(A)
\end{array}\right)
$$

with $A \in \mathfrak{s o}(4), \nu: \mathfrak{s o}(4) \rightarrow \mathfrak{s o}(3)$ and $B \in \mathfrak{p}$. The symmetries of $\mathfrak{p}$ and the map $\nu$ are explicitly defined by A.1

\section{ACKNowledgement}

The authors would like to thank Chuu-Lian Terng for useful conversations and for suggesting the problems addressed in this article.

\section{REFERENCES}

[1] M. Brück, X. Du, J. Park, and C.-L. Terng, The submanifold geometries associated to Grassmannian systems, Mem. Amer. Math. Soc. 155 (2002), no. 735, viii+95. MR.1875645 (2002k:53103) 
[2] F.E. Burstall, Isothermic surfaces: Conformal geometry, Clifford algebras and integrable systems, Integrable systems, geometry, and topology, AMS/IP Stud. Adv. Math., vol. 36, Amer. Math. Soc., Providence, RI, 2006, pp. 1-82. MR2222512 (2008b:53006)

[3] F.E. Burstall and M.A. Guest, Harmonic two-spheres in compact symmetric spaces, revisited, Math. Ann. 309 (1997), no. 4, 541-572. MR1483823 (99f:58046)

[4] F.E. Burstall and F. Pedit, Harmonic maps via Adler-Kostant-Symes theory, Harmonic maps and integrable systems, Aspects Math., E23, Vieweg, Braunschweig, 1994, pp. 221272. MR.1264189

[5] B. Dai and C.-L. Terng, Bäcklund transformations, Ward solitons, and unitons, J. Differential Geom. 75 (2007), no. 1, 57-108. MR.2282725 (2008h:58027)

[6] N.M. Donaldson and C.-L. Terng, Conformally flat submanifolds in spheres and integrable systems, 2007, Eprint: arXiv:math/0803.2754v2, 2008.

[7] N.M. Donaldson, Symmetric r-spaces: Submanifold geometry and Transformation theory, Ph.D. thesis, University of Bath, 2006.

[8] D. Ferus and F. Pedit, Isometric immersions of space forms and soliton theory, Math. Ann. 305 (1996), no. 2, 329-342. MR.1391218 (97d:53061)

[9] R. Harvey and H.B. Lawson, Jr., Calibrated geometries, Acta Math. 148 (1982), 47-157. MR666108 (85i:53058)

[10] A. Pressley and G. Segal, Loop groups, Oxford Mathematical Monographs, The Clarendon Press Oxford University Press, New York, 1986, Oxford Science Publications. MR900587 (88i:22049)

[11] C.-L. Terng, Soliton equations and differential geometry, J. Differential Geom 45 (1997), no. 2, 407-445. MR 1449979 (98e:58102)

[12] C.-L. Terng and K. Uhlenbeck, Bäcklund transformations and loop group actions, Comm. Pure Appl. Math. 53 (2000), no. 1, 1-75. MR.1715533 (2000k:37116)

[13] C.-L. Terng and E. Wang, Transformations of flat Lagrangian immersions and Egoroff nets, Asian J. Math. 12 (2008), no. 1, 99-119. MR2415015 (2009e:53077)

[14] K. Uhlenbeck, Harmonic maps into Lie groups: Classical solutions of the chiral model, J. Differential Geom. 30 (1989), no. 1, 1-50. MR1001271 (90g:58028)

Department of Mathematics, University of California, Irvine, Irvine, California 92697

E-mail address: ndonalds@math.uci.edu

Mathematics Institute, University of Oxford, 24-29 St Giles', Oxford, OX1 3LB, UNITED KINGDOM

E-mail address: foxd@maths.ox.ac.uk

Mathematisches Institut, Universität zu Köln, Weyertal 86-90, 50931 Köln, Germany

E-mail address: ogoertsc@math.uni-koeln.de 